\section{FRI0224 RITUXIMAB SELECTIVELY REDUCES IGG4 LEVELS IN RHEUMATOID ARTHRITIS PATIENTS}

A. Sarantopoulos, I. Gkougkourelas, M. Mytilinaiou, P. Klonizakis, A. Georgiadou, P. Boura. Clinical Immunology Unit, 2nd Dpt of Internal Medicine, Aristotle University of Thessaloniki, Thessaloniki, Greece

Background: Rituximab has been applied as a therapeutic strategy in a variety of diseases, including Rheumatoid Arthritis (RA) and IgG4-Related Disease $(\operatorname{lgG} 4-R D)^{1}$. On IgG4-RD, it has been shown that apart from B-cell depletion, rituximab induces remission by reducing IgG4 levels ${ }^{2}$.

Objectives: On this regard, we investigated weather B-cell depletion in RA is also associated with a selective reduction of any lgG subclass, especially lgG4.

Methods: 31 RA patients, 25/6 female/male, median age 59 years (34-73), duration of disease 9,5 years (1-30) on standard of care DMARD treatment and rituximab administration every 6 months for 2 years were investigated for alterations on disease activity along with Igs' and IgG subclasses levels. All parameters were assessed at enrollment (T0), and after 6, 12 and 24 months. On this 2-year period all patients had been periodically receiving rituximab every 6 months.

Results: After 2 years of rituximab administration, patients achieved a good response to treatment (EULAR criteria). Igs' levels were not statistically altered, though all of them declined (data for IgM and IgA not shown). Furthermore, from IgG subclasses, only lgG4 levels statistically declined.

Conclusions: This is the first time that lgG4 variations are investigated in a nonIgG4RD after rituximab administration. Our results imply that IgG4 may be actively implicated in RA pathophysiology, since disease remission is accompanied by only IgG4 level reduction among all classes and subclasses of Igs'. Furthermore, in RA patients, rituximab may exert its therapeutic results not only via B-cell depletion, but also via IgG4 levels reduction.

References:

[1] Carruthers MN, Topazian MD, Khosroshahi A, et al. Rituximab for IgG4related disease: a prospective, open-label trial. Ann Rheum Dis. 2015 Jun;74(6):1171-7.

[2] Khosroshahi A, Bloch DB, Deshpande V, Stone JH. Rituximab Therapy Leads to Rapid Decline of Serum IgG4 Levels and Prompt Clinical Improvement in IgG4-Related Systemic Disease. Arthritis Rheum. 2010 Jun; 62(6): 1755-62. Disclosure of Interest: None declared

DOI: 10.1136/annrheumdis-2017-eular.6690

\section{FRI0225 ANEMIA IS A BETTER PREDICTOR FOR RADIOGRAPHIC DAMAGE IN RHEUMATOID ARTHRITIS THAN DAS28 WHEN DETERMINED BEFORE START OF TOCILIZUMAB-TREATMENT - A SECONDARY ANALYSIS FROM THE ACT-RAY TRIAL}

B. Möller ${ }^{1}$, G. Scholz ${ }^{1}$, M. Dougados ${ }^{2}$, T. Huizinga ${ }^{3}$, P.M. Villiger ${ }^{1}$, A. Finckh ${ }^{4}$ ${ }^{1}$ Rheumatology, Immunology \& Allergology, Inselspital - Bern University Hospital, Bern, Switzerland; ${ }^{2}$ Rheumatology, Hôpital Cochin, Paris, France;

${ }^{3}$ Rheumatology, Leiden University Medical Center, Leiden, Netherlands;

${ }^{4}$ Rheumatology, University Hospital Geneva, Geneva, Switzerland

Background: Clinical remission, or at least low disease activity, as measured by DAS28 or alternative compound indices is currently the goal of RA treatment. Anemia in the context of RA is mainly driven by tumor necrosis factor alpha (TNF- $\alpha$ ) and interleukin-six (IL-6), and may serve as a simple biological marker of inflammation. Anemia was recently discovered as a largely DAS28independent parameter to predict radiographic damage progression in RA, both with conventional synthetic disease-modifying antirheumatic drugs (csDMARDs) or with anti-TNF agents.

Objectives: To study the predictive value of anemia in relation to DAS28 for radiographically detectable joint damage progression in patients treated with IL-6R inhibitor tocilizumab (TCZ) plus conventional synthetic disease-modifying antirheumatic drugs (csDMARDs) when used in a treat to target concept.

Methods: In the ACT-RAY trial, all patients were methotrexate (MTX) inadequate responders and randomized to receive either TCZ plus MTX (add-on) or TCZ plus placebo (switch strategy) for at least one year. After week 24, open-label csDMARDs other than MTX could be added according to a treat-to-target approach in patients with moderate or high disease activity (DAS28 $>3.2$ ). After week 52, patients in sustained clinical remission (DAS28<2.6) could discontinue TCZ before stopping csDMARDs, and finally MTX/Placebo. The exposure of interest was anemia, defined using the WHO definition or the more liberal NHANES definition, both at baseline and over time. The primary outcome was the rate of joint damage progression measured by the change in the modified total Sharp score $(\triangle \mathrm{mTSS})$. We used longitudinal multivariate mixed effects models to test the impact of anemia on $\Delta$ mTSS. To examine the association of anemia to $\triangle \mathrm{mTSS}$ independently from RA disease activity, the models were run with and without DAS28 at baseline and DAS28 over time.

Results: Of 556 randomised patients, complete datasets for fully adjusted models were available from 285 patients. Overall radiographic progression was regarded to be minimal, with insignificant differences in favor of the add-on strategy. Median annual $\triangle$ mTSS before inclusion was 2.9 (IQR 1.5 to 6.4 ) in patients without and 5.5 (IQR 2.7 to 11.1) in patients with baseline anemia, but evolved subsequently similar when being on TCZ. Anemia at baseline was a strong predictor of mTSS (per WHO definition: coefficient 14.8, 95\% Cl 7.6-21.9, $p<0.001$; per NHANES definition: coefficient $14.695 \% \mathrm{Cl} 7.6-21.5 \mathrm{p}<0.001$ ), as well as baseline DAS28 (coefficient $3.9,95 \% \mathrm{Cl} 0.7-7.0, p=0.016)$. Mean DAS28 over time $(p<0.001)$, in contrast to anemia data over time when obtained in patients already on TCZ, was significantly associated with subsequent $\triangle \mathrm{mTSS}$. Baseline anemia in contrast to baseline DAS28 remained a significant predictor of mTSS for up to two years on TCZ in fully adjusted multivariate analyses $(p<0.05)$, including time-variant DAS28.

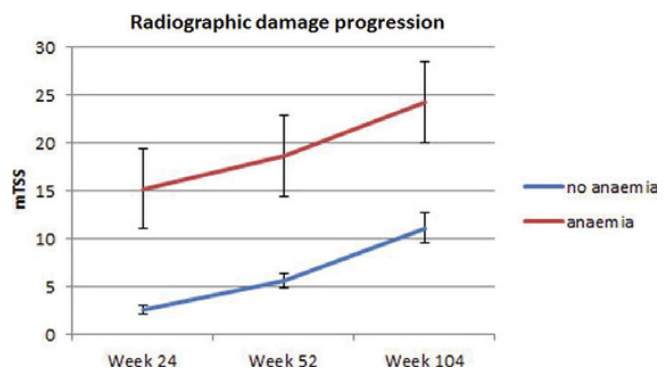

Conclusions: Anemia may be a strong and DAS28-independent long-term predictor of radiographic joint damage progression. Present data from patients on IL-6R blockade, a potent target-specific RA treatment with major impact also on erythropoiesis, add importantly to the growing body of evidence for the studied association.

Disclosure of Interest: None declared

DOI: 10.1136/annrheumdis-2017-eular.6685

\section{FRI0226 RITUXIMAB IN RHEUMATOID ARTHRITIS WITH INTERSTITIAL LUNG DISEASE: A MULTICENTER STUDY}

C. Fernández-Díaz ${ }^{1}$, M. Martin-Lopez ${ }^{2}$, M. Carrasco-Cubero ${ }^{3}$, D. Reina-Sanz ${ }^{4}$, P. Rubio-Muñoz ${ }^{5}$, A. Urruticoechea-Arana ${ }^{6}$, J. Miranda-Filloy ${ }^{7}$,

N. Vegas-Revenga ${ }^{1}$, L. Dominguez-Casas ${ }^{1}$, M. Gonzalez-Gay ${ }^{1}$, R. Blanco ${ }^{1}$.

${ }^{1}$ Rheumatology, Hospital Universitario Marques de Valdecilla, Santander;

${ }^{2}$ Rheumatology, Hospital Universitario 12 de Octubre, Madrid; ${ }^{3}$ Rheumatology, Hospital Infanta Cristina, Badajoz; ${ }^{4}$ Rheumatology, Hospital Sant Joan Despi

Moises Boggi; ${ }^{5}$ Rheumatology, Hospital Universitario Germans Trias i Pujol,

Barcelona; ${ }^{6}$ Rheumatology, Hospital Can Misses, Ibiza; ${ }^{7}$ Rheumatology, Hospital Universitario Lucus Augusti, Lugo, Spain

Background: Anti-TNF $\alpha$ drugs and several conventional disease-modifying antirheumatic drugs (DMARDs) such as methotrexate (MTX) have been involved in the development of Interstitial Lung Disease (ILD).

Objectives: Our aim was to assess the efficacy and safety of Rituximab (RTX) in RA patients with ILD.

Methods: Multicenter study of RA patients with ILD treated with RTX. ILD was diagnosed by high-resolution computed tomography (HRCT). RTX was used at standard dose (1 gx2 and premedication with iv Methylprednisolone for a six month interval. We assess the the following variables: a) 1-point change in the degree of dyspnea according to the Modified Medical Research Council (MMRC); b) FVC improvement $\geq 10 \%$; and improvement $\geq 10 \%$ in DLCO; c) radiological changes in HRCT scan, and d) changes in the joint assessment measured by DAS28 score.

Results: We studied 18 patients ( 13 women $/ 5$ men) with ILD associated to RA. The mean age $\pm S D$ was $62.8 \pm 11.0$ years. The median [IQR] to progression of RA was 5.25 [2-12.8] years. They had received the following DMARDs previously; MTX $(n=11)$, Leflunomide (LFN) $(n=9)$ mycophenolate (MMF) $(n=1)$ sulfasalazine (SSZ) $(n=5)$, hydroxichloroquine (HCQ) $(n=4)$, azathioprine $(A Z A)(n=1)$, gold salts $(n=1)$, D-penicillamine $(n=1)$, cyclophosphamide $(n=1) .7$ patients had previously received biological drugs. RA was seropositive in 16 cases (89\%). Besides HRCT, the diagnosis of ILD was confirmed by biopsy in 4 patients. In 2 patients ILD was drug-related: MTX $(n=2)$. RTX was prescribed as monotherapy $(n=7)$ and combined with DMARDs (11. The DMARDs prescribed were: LFD (4), SSZ (2),

Abstract FRI0224 - Table 1. DAS28 and IgG class and subclasses variations. Because of non-normal distribution of our sample, the results were expressed as median and range and statistical analysis was performed by using the Kruskal Wallis tests

\begin{tabular}{|c|c|c|c|c|c|c|}
\hline & DAS28 & $\lg G$ & $\lg \mathrm{g} 1$ & $\lg G 2$ & $\operatorname{lgG} 3$ & $\operatorname{lgG} 4$ \\
\hline T0 & $4,46(2,06-6)$ * & $12,2(5,1-23,1)$ & $7,7(3,59-12,4)$ & $2,7(0,844-5,21)$ & $0,573(0,079-1,85)$ & $0,451(0,032-2,1)^{\star}$ \\
\hline T6 & $3,8(2,2-6,44)$ & $11,1(5,78-15)$ & $7,28(3,38-11,2)$ & $2,7(1,23-5,36)$ & $0,445(0,086-1,58)$ & $0,35(0,044-1,06)$ \\
\hline T12 & $4,01(1,68-5,77)$ & $11,1(5,36-19,2)$ & $6,81(3,35-12,2)$ & $2,53(0,876-4,67)$ & $0,423(0,07-1,88)$ & $0,279(0,036-1,24)$ \\
\hline T24 & $\begin{array}{c}3,27(1,5-5,07) * \\
{ }^{*} \mathrm{p}<0.05\end{array}$ & $11,2(4,92-14,5)$ & $6,59(3,34-10,3)$ & $2,42(0,92-4,7)$ & $0,405(0,08-0,823)$ & $\begin{array}{c}0,248(0,025-1,08)^{*} \\
{ }^{*} p<0.05\end{array}$ \\
\hline
\end{tabular}


MTX (3), HCQ (1), AZA (1) MMF (1). A significant improvement of the dyspnea was observed. FVC and HRCT showed an improvement in the period between 6 and 12 months. DLCO remained stable in the majority of the patients (\%). DAS28 also improved.

After a follow-up of 12 months, the only serious adverse effect was a severe Infection respiratory.

Table 1

\begin{tabular}{lcccc}
\hline & Baseline & 3 months & 6 months & 12 months \\
\hline MMRC, $n$ (\%) & 18 & 15 & 16 & 16 \\
- No change & & $10(67)$ & $10(63)$ & $10(63)$ \\
- Improvement & & $5(33)$ & $6(37)$ & $6(37)$ \\
- Worsening & & 0 & 0 & 0 \\
CVF, n (\%) & 16 & 10 & 5 & 13 \\
- No change & & $7(70)$ & $5(100)$ & $10(77)$ \\
- Improvement & & $1(10)$ & 0 & $3(23)$ \\
- Worsening & & $2(20)$ & 0 & 0 \\
DLCO, n (\%) & 14 & 6 & 3 & 12 \\
- No change & & $5(83)$ & $1(33)$ & $8(66)$ \\
- Improvement & & 0 & $2(67) 72(17)$ & \\
- Worsening & & $1(17)$ & 0 & $2(17)$ \\
HRCT, n (\%) & & 7 & 9 & 7 \\
- No change & & $6(86)$ & $7(78)$ & $4(57)$ \\
- Improvement & & $1(14)$ & $1(11)$ & $3(43)$ \\
- Worsening & & 0 & $1(11)$ & 0 \\
JOINT, DAS28 - Mean & $4.32 \pm 1.35$ & $3,21 \pm 0.73$ & $3,44 \pm 0.87$ & $2.83 \pm 0.70$ \\
CRP (mg/dl)-, Mean & $2.01 \pm 2.35$ & $1.12 \pm 0.9$ & $1.98 \pm 1.95$ & $1.03 \pm 0.9$ \\
ESR (mm/1sth), Mean & $49.0 \pm 30.64$ & $37.79 \pm 37,73$ & $38.63 \pm 30.63$ & $24.50 \pm 22$ \\
\hline & & & & \\
& & & & \\
& & & &
\end{tabular}

Conclusions: RTX seems to be an effective and relatively safe treatment in RA patients with ILD. However, these data should be verified in prospective and randomized studies.

Disclosure of Interest: None declared

DOI: 10.1136/annrheumdis-2017-eular.3275

\section{FRI0227 SARILUMAB SIGNIFICANTLY SUPPRESSES CIRCULATING BIOMARKERS OF BONE RESORPTION AND CARDIOVASCULAR RISK COMPARED WITH ADALIMUMAB: BIOMARKER ANALYSIS FROM THE PHASE 3 MONARCH STUDY}

C. Gabay ${ }^{1}$, J. Msihid ${ }^{2}$, C. Paccard ${ }^{2}$, M. Zilberstein ${ }^{3}$, N.M. Graham ${ }^{4}$, A. Boyapati 4 . ${ }^{1}$ University Hospitals of Geneva, Geneva, Switzerland; ${ }^{2}$ Sanofi R\&D, Chilly-Mazarin, France; ${ }^{3}$ Sanofi R\&D, Bridgewater; ${ }^{4}$ Regeneron Pharmaceuticals, Inc, Tarrytown, United States

Background: MONARCH (NCT02332590) was a randomized, active-controlled, double-blind, double-dummy, phase 3 superiority trial comparing sarilumab monotherapy with adalimumab monotherapy. Exploratory biomarkers associated with inflammation, bone erosion, and cardiovascular (CV) risk were evaluated in this study.

Objectives: To compare the effects of sarilumab monotherapy vs adalimumab monotherapy on circulating biomarkers associated with acute-phase response (CRP, serum amyloid A [SAA]), bone resorption (RANKL and osteoprotegerin [OPG]), and CV risk (lipoprotein (a) [Lp(a)]) in patients from MONARCH.

Methods: Sera were analyzed at baseline and posttreatment through wk 24 from patients who consented to biomarker analyses and received SC sarilumab $200 \mathrm{mg} \mathrm{q} 2 \mathrm{w}(\mathrm{N}=153)$ or adalimumab $40 \mathrm{mg}$ q2w $(\mathrm{N}=154)$. Biomarkers were assessed using validated ELISAs. Nonparametric methods were used to evaluate differences in the percent change from baseline in biomarker levels between treatments at each time point. Percent change from baseline in biomarkers at wk 24 was also compared, separately by treatment group, between ACR50 responders and nonresponders at wk 24. The Benjamini-Hochberg procedure was used to correct $P$ values for multiplicity and control false discovery rate. Significance level was $P<0.05$.

Results: A significant difference in RANKL was observed at wks 2 and 24 between sarilumab and adalimumab groups $(P<0.0001$; Table). Numerically, RANKL decreased after sarilumab and increased after adalimumab treatment. Significantly greater reductions in $\mathrm{Lp}(\mathrm{a}), \mathrm{SAA}$, and CRP were observed at wks 12 and 24 after treatment with sarilumab vs adalimumab. The difference in OPG between groups was significant at wk 2 only.

Table 1. Median Percent Change From Baseline in Serum Concentrations of Circulating Biomarkers

\begin{tabular}{llcc}
\hline & & Sarilumab 200 mg q2w $(\mathrm{N}=153)$ & Adalimumab 40 mg q2w $(\mathrm{N}=154)$ \\
\hline CRP & Wk 12 & $-94.2^{\dagger}$ & -29.7 \\
& Wk 24 & $-94.0^{\dagger}$ & -24.0 \\
SAA & Wk 12 & $-79.9^{\dagger}$ & -32.2 \\
& Wk 24 & $-83.2^{\dagger}$ & -17.4 \\
RANKL & Wk 2 & $-2.5^{\dagger}$ & 4.4 \\
& Wk 24 & $-18.3^{\dagger}$ & 10.5 \\
OPG & Wk 2 & $0.9^{\star}$ & -4.0 \\
& Wk 24 & 2.2 & 3.3 \\
Lp(a) & Wk 12 & $-35.0^{\dagger}$ & -0.4 \\
& Wk 24 & $-41.0^{\dagger}$ & -2.8 \\
\hline
\end{tabular}

${ }^{\star}$ Adjusted $P<0.01$ vs adalimumab. ${ }^{\dagger}$ Adjusted $P<0.0001$ vs adalimumab.
At wk 24, change in OPG, RANKL, and Lp(a) did not differ between ACR50 responders and nonresponders at wk 24 in either treatment group. Responders in both groups demonstrated greater reductions vs nonresponders in CRP (sarilumab, $-95.8 \%$ vs $-87.3 \%$; adalimumab, $-47.6 \%$ vs $-6.4 \%$; all $P<0.01$ ); a trend was observed for SAA (sarilumab, $-92.3 \%$ vs $-73.2 \%$; adalimumab, $-33.0 \%$ vs $0.0 \%$; unadjusted $P<0.05$, not significant after adjustment).

Conclusions: Sarilumab monotherapy significantly suppressed bone-resorptive and $\mathrm{CV}$ risk markers to a greater degree than adalimumab monotherapy. Reductions in CRP were significantly different in ACR50 responders vs nonresponders after either treatment. Analyses to assess predictive and prognostic effects of biomarkers (including markers of myeloid and lymphoid synovial phenotypes) are ongoing.

Acknowledgements: This study was sponsored by Sanofi Genzyme and Regeneron Pharmaceuticals, Inc. Editorial support was provided by MedThink SciCom and funded by Sanofi Genzyme and Regeneron Pharmaceuticals, Inc.

Disclosure of Interest: C. Gabay Consultant for: Roche, Merck, AbbVie, Pfizer, Bristol-Myers Squibb, Sanofi-Aventis, and AB2 Bio, J. Msihid Shareholder of: Sanofi R\&D, Employee of: Sanofi R\&D, C. Paccard Shareholder of: Sanofi R\&D, Employee of: Sanofi R\&D, M. Zilberstein Shareholder of: Sanofi R\&D, Employee of: Sanofi R\&D, N. Graham Shareholder of: Regeneron Pharmaceuticals, Inc, Employee of: Regeneron Pharmaceuticals, Inc, A. Boyapati Shareholder of: Regeneron Pharmaceuticals, Inc, Employee of: Regeneron Pharmaceuticals, Inc DOI: 10.1136/annrheumdis-2017-eular.4534

\section{FRI0228 UGT1A1 GENETIC VARIANTS ARE ASSOCIATED WITH INCREASES IN BILIRUBIN LEVELS IN RHEUMATOID ARTHRITIS PATIENTS TREATED WITH SARILUMAB}

A. Damask ${ }^{1}$, A. Boyapati ${ }^{1}$, J.D. Hamilton ${ }^{1}$, S. Hamon ${ }^{1}$, C. Paccard ${ }^{2}$, J. Parrino ${ }^{1}$, J. van Adelsberg ${ }^{1}$, N.M. Graham ${ }^{1}$, J. Penn ${ }^{1}$, A. Lopez ${ }^{1}$, J. Reid ${ }^{1}$, J. Overton ${ }^{1}$,

A. Baras ${ }^{1}$, A.R. Shuldiner ${ }^{1}$, C. Paulding ${ }^{1} .{ }^{1}$ Regeneron Pharmaceuticals, Inc, Tarrytown, United States; ${ }^{2}$ Sanofi R\&D, Chilly-Mazarin, France

Background: Sarilumab is a human mAb that blocks IL-6 from binding to both membrane-bound and soluble IL-6R $\alpha$. Variants in the UGT1A1 gene have been shown to be strongly associated with increased unconjugated bilirubin levels in patients treated with tocilizumab, another IL-6R $\alpha$ inhibitor. ${ }^{1,2}$ UGT1A1 encodes the enzyme responsible for the glucuronidation of bilirubin and variation in this gene is also responsible for Gilbert's syndrome, a mild benign condition characterized by elevations in unconjugated bilirubin and jaundice. The underlying main genetic variation responsible for Gilbert's syndrome has been identified as a TA repeat located in the promoter of UGT1A1 (UGT1A1*28 allele), which is in linkage disequilibrium with a single nucleotide polymorphism, rs6742078, previously associated with higher bilirubin levels after tocilizumab treatment. ${ }^{1}$

Objectives: To test for an association between rs6742078 and bilirubin levels in RA patients treated with sarilumab.

Methods: DNA was collected from patients enrolled in MOBILITY (NCT01061736), which evaluated the efficacy and safety of sarilumab + methotrexate (MTX) in RA patients with inadequate response to MTX. The pharmacogenetic analysis was conducted in 599 Caucasian patients treated with MTX + sarilumab (150 or $200 \mathrm{mg} \mathrm{q2w}$ ) or placebo. Log-transformed unconjugated and total bilirubin levels were analyzed at baseline and over the treatment period (using maximum bilirubin).

Results: There was a strong association between the rs6742078 TT genotype and higher unconjugated bilirubin levels. The least squares mean (SE) for patients at baseline with the TT genotype was $0.48(0.02) \mathrm{mg} / \mathrm{dL}$ vs $0.25(0.009)$ and 0.21 $(0.009) \mathrm{mg} / \mathrm{dL}$ for those with GT and GG genotypes, respectively $\left(p=1.02 \times 10^{-21}\right)$. After sarilumab treatment, the difference between genotype groups increased over the course of the study $\left(p=4.3 \times 10^{-10} ;\right.$ Figure). In the binary analysis of maximum total bilirubin in sarilumab-treated patients, the TT genotype was significantly associated with mild bilirubin elevations ( $\mathrm{OR}=34.7 ; p=1.2 \times 10^{-8}$; Table).

Table 1. Maximum Total Bilirubin in Sarilumab-Treated Patients by rs6742078 Genotype

\begin{tabular}{lccc}
\hline & \multicolumn{3}{c}{ Maximum total bilirubin, $\mathrm{n}(\%)$} \\
\cline { 2 - 4 } Genotype, $\mathrm{n}(\%)$ & $\leq 1.5 \times \mathrm{ULN}$ & $>1.5 \times \mathrm{ULN}$ & Total \\
\hline GG/GT & $352(92)$ & $4(27)$ & $356(90)$ \\
TT & $29(8)$ & $11(73)^{\mathrm{a}}$ & $40(10)$ \\
& 381 & 15 & 396
\end{tabular}

$\mathrm{OR}=34.7^{\mathrm{b}} ; p=1.2 \times 10^{-8} .{ }^{\text {a Elevations remained } \leq 2 \times \mathrm{ULN} \text {. }{ }^{\mathrm{b}} \text { Logistic regression with recessive ge- }}$ netic model, adjusting for ancestry covariates.

Conclusions: The association observed between the rs6742078 TT genotype in UGT1A1 and unconjugated bilirubin elevations in sarilumab-treated patients is consistent with previous observations in tocilizumab-treated patients. These findings suggest that sarilumab-related increases in bilirubin levels are likely benign and caused by common genetic variation in UGT1A1 and are not due to underlying liver injury.

References:

[1] Mori et al. Mod Rheumatol. 2012;22:515-523.

[2] Lee et al. Pharmacogenet Genomics. 2011;21:365-374

Acknowledgements: This study was sponsored by Sanofi Genzyme and Regeneron Pharmaceuticals, Inc. Editorial support was provided by MedThink SciCom and funded by Sanofi Genzyme and Regeneron Pharmaceuticals, Inc. 